\title{
Application of Judicial Case Method on Health Law Teaching
}

\author{
Lin Yang ${ }^{1, a}$ and Xuan Zheng ${ }^{1, b^{*}}$ \\ ${ }^{1}$ Institute of Medicine and Nursing, Hubei University of Medicine, Shiyan, China \\ a5810030@qq.com, b826725887@ qq.com \\ *The corresponding author
}

Keywords: Judicial case; Health law; Judicial case teaching method; Case teaching method

\begin{abstract}
As a compulsory course for medical students, health law has mature teaching methods. Case teaching methods in the teaching of health law have been commonly used. However, most of the implementation of case teaching methods remains at the stage of "illustrate", "case teaching", which may not reflect the true essence of case teaching method. Judicial case teaching method emphasizes on the reduction of real cases in trial processes. It pays attention to the skill training of health law. This paper focuses on the teaching reform of health law, mainly from the perspective of the judicial case teaching method. The specific implementation plan proposed here unifies the real cases in our country and judicial case teaching method to achieve a better teaching effect.
\end{abstract}

\section{Acknowledgement}

This research was financially supported by the 2013 Research Project of Higher Education Reform in Hubei Province (2013B242).

\section{判例教学法在我国卫生法教学中的应用研究}

\author{
杨琳 $1^{1, \mathrm{a}}$, 郑玄 $1^{1, \mathrm{~b}}$ \\ 1. 湖北医药学院 药护学院, 中国 湖北 十堰 442000 \\ a5810030@qq.com, b26725887@qq.com
}

摘要: 卫生法作为医学相关专业的必修课程在教学方法上已趋向成熟, 案例教学法在卫生法 教学中已被普遍适用, 但实施中案例教学多停留于 “举例说明”、“事例教学” 等形式, 并未 真正体现出判例法国家判例教学的精髓。判例教学法强调对真实案件审理过程的还原, 注重 对学生的卫生法律技能培养。文章主要从判例教学法的视角探讨卫生法的教学改革, 结合我 国实际重点探讨判例教学法在卫生法教学中的具体实施方案, 以追求更好的教学效果。

\section{关键词：判例; 卫生法; 判例教学法; 案例教学法}

\section{1. 引言}

近几年来, 随着法治国家建设进程的加快, 卫生法领域的各项立法得以不断充实、完善, 卫 生法这门学科也得到长足的发展, 已成为医学高等教育的必修课程, 且教学内容、教学方法 也逐渐成熟。结合医学相关专业的特点, 卫生法的教学尤其注重运用法学理论和方法解决医 学实践中的实际问题。但在卫生法的实际教学过程中, 案例教学方法却多流于形式, 对于诸 多案例学生多知其然却不知其所以然, 分析能力并未如预期般得到普遍提升。当然师资情况、 教学方法等都会影响最终的教学效果, 本文则主要以判例教学法的视角来探讨卫生法的教学 改革。 


\section{2. 判例教学法的内涵}

2.1. 判例教学法的由来

判例教学法源起于英美等普通法系国家。美国式法学判例教学法常常被具有浓郁大陆法传统 的中国法律人作为矫正讲授式教学模式和立法与理论本本主义的重要手段 [1]。判例教学法最 早是在 1860-1870 年代纽约大学波默罗伊教授 (Pomeroy) 讲授 “衡平理论” 时用过 [2], 并从哈 佛大学兰德尔 (Langde11) 教授开始制度化 [3]。判例教学法强调学生思维能力的训练, 通过 这种方式培养学生的法律意识和法律观念, 从而打破纯理论性讲授的教条主义授课模式, 让 书本理论和实践操作结合起来。作为英美法系国家法学教育的基本方法, 判例教学形式主要 是以司法判例即真实案件的判决为中心，结合苏格拉底问答式教学方法进行。例如，美国法 学院的教科书被称为 “判例汇编”, 其内容包括围绕判例的注释、问题文章、参考文献等, 教 学与考试也主要是针对判例所进行的讨论、辩论、分析、评价 [4]。

2.2. 判例教学法和案例教学法的区分

案例教学法一般被界定为通过对一个具体教育情境的描述, 引导学生对这些特殊情境进行讨 论的一种教学方法。从本质上看, 案例教学即由判例教学转化而来。在法学教育中一直强调 对学生的法律实务教育, 案例教学法即满足了这一教学要求。但是, 我国现行的 “法律案例 教学法” 是在一个泛指的意义上使用的, 没有区分 “举例教学” 和 “案件教学”、“事例教学” 和 “判例教学” 等相关的案例教学形式和范畴 [5]。在卫生法实际的教学中, 为了方便授课需 要, 加强案例和相关知识点的联结程度, 教师一般会挑选、整理、甚至重新编写案例。案例 的编写是对真实案例进行细节上的改编或整体上的重新设计, 通过对原始材料的加工, 重新 呈现的案例知识点可能更为集中, 也可能综合性更强 [6]。即案例教学强调和案例与教学内容 的相关性。而判例教学法则强调对真实案件审理过程的还原, 通过研究判例模拟当事人、律 师、法官等角色运用法律思维去思考、分析、解决问题; 甚至还期待通过判例培养学生在证 据审核、信息获取、沟通协调、谈判辩论乃至制定规则等方面的技能。可见，判例教学法更 注重对学生全方位的法律技能培养。

2.3. 判例教学法的优势

案例教学法的使用大多停留在 “举例” 的层面，讲授思路通常体现为 “案件介绍一一提问一 一答案” 模式。困境就在于没有紧贴司法现实, 更多的是重视法律文本和学者的抽象阐述, 注重法律的教义分析, 而不太注重个案及司法判决, 缺乏对具体案件的法律问题的严格分析 训练 [7]。判例教学法则直接将判例作为一种教学工具, 判例中不但有判决结果, 还有原被告 的意见、法官的推理及法律理由, 这可以使学生更清楚地意识到源于卫生实践中法律的力量, 并尽量发掘判例及其背后的故事，建立对司法审理过程及判决结果的正确认识。这种教学方 式能够缩短法律理论与司法实务的差距, 让学生熟悉最新的法条内容和司法运作方式, 在教 学效果方面更能锻炼学生的法律思维能力和实际运用能力。判例的形象性、生动性、写实性 也可以帮助学生有效地理解卫生法的精髓, 有助于提高学生对卫生法律规范的理解能力。

\section{3. 判例教学法使用时应注意的问题}

\section{1. 注意对学生思维方式的引导}

卫生法是一门医学、卫生学和法学融合的交叉学科, 交叉学科研究的复杂性远远高于单一学 科的研究, 如何将不同学科的资源有效组织起来, 为了共同目标协同工作是开展交叉学科研 究的关键问题 [8]。因此这种边缘学科在开展研究时对教师和学生要求都比较高, 例如关于医 疗纠纷的判例通常会涉及临床专业技能、医疗专业鉴定、举证负担、过错认定、法理分析、 侵权责任理论等多方面的内容, 这就要求教师必须给予适当的引导, 从不同角度指引学生思 考, 既可以拓宽学生的知识面, 也可以重点突出相关理论, 并能让学生在自身的专业基础上 有更深层次的思考。另外, 在判例中存在多方主体, 这也要求教师要从不同主体的角度引导 学生对案件进行多元化的思维, 从当事人、律师、法官或关系人、公众的不同视角来培养学 
生独立思考、全面分析的能力。

\section{2. 注意提高学生在课堂上的参与度}

判例法教学旨在打破传统的填鸭式教学方法, 从以 “教” 为主的教学模式转变为以 “学” 为 主教学模式。在传统卫生法学教学中, 教师具有绝对的权威, 学生对教师要心存敬畏, 这种 权力结构严重影响了师生之间的平等交流和沟通 [9]。因此在运用判例教学法时, 教师首先须 和学生平等地交流、沟通, 从学生的认知出发, 营造一个平等、和谐的课堂环境; 进而让学 生主动、积极地参与到判例教学过程中来, 对案例进行独立的思考、讨论和分析, 教师只是 从旁加以引导和必要的调控、指正。当然, 要想让学生更好地参与到判例教学过程, 必须辅 之以必要的课前准备和课下自主学习, 这就需要教师根据学生的水平、能力在教学中进行适 当的安排。

3. 3. 注意引导学生正确看待司法判例

判例法教学所使用的都是真实的司法判例, 基本都由法院经过审理做出了终审判决, 司法过 程要求 “以事实为根据, 以法律为准绳”, 但做一个司法判决不同于做一道数学题。“纸面上” 的法律条文规定要转化为具体的司法判决, 无疑是一个既具有高度技术性又具有实践智慧性 的过程 [10]。在卫生法的判例教学中, 很多案例无疑都和医疗纠纷有关, 医方是否有过错、 责任大小等关键争议问题需要通过鉴定、举证等多项程序来加以认定, 结果可能不是那么尽 善尽美。判例教学注重对学生思维的拓展和训练, 教师应当允许学生对判例有不同的观点, 对判例开展批判性思考和探讨, 但也应当引导学生客观、正确地看待这些判例, 尊重法律的 权威性。

3. 4. 判例教学法并不排斥案例教学法

教学方法存在多样性, 不同的教学方法互补互助才能实现最佳的教学效果。多元化的教学模 式能为培养学生的法律人格提供真实的实践场所 [11]。案例教学法在卫生法教学中长期使用 且在不断地改革, 可见案例教学有其自身的特色和价值。判例教学法的使用意在充实、完善 卫生法的教学方法, 弥补其他教学方法的短板, 并不排斥其他教学方法的使用。相反, 判例 教学和案例教学、法律诊所教学、PBL 等其他教学方法应当有效结合起来, 教师应当根据教 学内容适当调整教学方法, 才能达到更好的教学效果。

\section{4. 判例教学法在卫生法教学中的具体方案设计}

4.1. 判例素材的选择

我国虽然不像英美法系国家那样明确肯定判例的法律沸源地位，但最高人民法院公布出的“指 导性案例” 是具备一定的参考价值的, 且这些案例通常具备一定的典型示范作用, 其中卫生 法领域的相关案例无疑是比较可靠、权威的判例资源。同时, 随着司法公开的改革, 现在所 有的公开审理案件判决结果都要求对外公开, 在各级法院的官网上都可以查阅到判决书, 这 就成为卫生法教学中最为丰富的判例来源。此外, 判例教学也可以选择一些尚未定论的诉讼 案件, 只要其具备教学价值就可以引入教学, 这些案件无疑能够激发出学生更广的思考空间。 4.2. 教学组织与安排

如前所述, 判例教学法中应当坚持以学生为中心, 这就意味着必须尽量缩减课堂上教师理论 讲授的时间。而学生要想在课堂上充分地讨论, 并适当扩展、深化, 就必须在课前对判例有 一定的了解和准备, 因此, 卫生法的判例教学具体实施应包含两个环节, 即课前准备和课堂 教学。

\subsection{1 课前准备阶段}

判例教学一般选择的都是已经经过法院审理产生判决的案件, 内容丰富, 信息量大, 这就要 求教师应在课堂教学之前将相关的判例资料交给学生, 根据案件的复杂程度来决定提前 1 周 或 2 周, 让学生有充分的时间对判例进行了解和思考; 同时, 教师也可以配合判例提前设计 和教学内容相关的思考题, 让学生现行思考, 以方便在课堂上能够展开有效的讨论。 


\subsection{2 课堂教学阶段}

卫生法授课多针对临床、护理等医学相关专业, 且大班授课, 班级人数偏多, 因此教师可提 前对学生编组, 分期进行判例的准备。课堂上, 由负责该期判例的小组通过 PPT 展示或角色 扮演等方式, 提出问题并给出解决方案设计, 再由其他同学提问或表达不同观点, 进行参与 式讨论。期间教师应及时纠正学生的错误, 讨论结束后教师应作陈述性发言, 梳理该课的基 本知识点, 并给予必要的讲解。

4.3. 判例教学应纳入学生考评机制

目前高校对学生在考评方面多在尝试建立多层次的考核与评价体系, 构建反应层、参与层、 结果层等多层次评价结构, 通过多维考核, 促进学生全面发展 [12]。判例教学使得学生付出 更多的时间查阅资料并思考, 这种努力应当纳入学生的考评机制。具体操作上, 可结合学生 的表现给出小组成绩、个人成绩等课堂成绩, 再结合各校、各专业的具体情况安排平时成绩、 课堂成绩、期末成绩在最后总成绩中的比例。

\section{5. 结语}

它山之石, 可以攻玉。判例教学法起源于英美法系国家的法学教育, 目前也主要盛行于判例 法国家。我国在卫生法教学中已经转变了教学理念, 进行了一定的教学改革, 但是, 当下的 卫生法教学却恰恰缺少一个将理论学习与实践操作结合起来进行训练的中间环节。判例法教 学虽不能说能完全解决教学中的问题, 但至少能够一定程度上弥补当下卫生法教学的不足, 提高教学效率, 达成更好的教学效果。

\section{6. 致谢}

本文系湖北省教育科学 “十二五”规划 2013 年度立项课题成果 (2013B242)。

\section{参考文献}

[1] 万猛, 李晓辉. 问解案例教学法 $[J]$. 中国大学教学, 2014, 3:73-79.

[2] Robert Stevens. Law School: Legal Education in America from the1850s to the 1980s [M]. New Jersey: The Law Book Exchance, 2001: 68.

[3] Bruce A. Kimball, Young Chiristopher Langdell, 1826-1854: Theformation of an Educational Reformer [J]. Legal education, 2002, 56(1):189.

[4] 峗怡, 贺加. 判例教学法对我国卫生法教学改革的重塑与推进 $[J]$. 医学与哲学 (人文社 会医学版)，2010，31(1):63-64.

[5] 唐东楚. 论法律案例教学中 “判例” 的地位和作用 $[J]$. 湖南省政法管理干部学院学报, 2002, 18(5) : 108-109.

[6] 任为民, 案例教学法在医事法教学中的应用 $[\mathrm{J}]$. 中国科教创新导刊, 2009，31：80.

[7] 苏力. 中国法律技能教育的制度分析 [J]．法学家，2008(2)：35.

[8] 孙丽珍, 王淑琴等. 研究型大学交叉学科研究的组织模式与载体分析 [J]. 研究与发展管 理, 2013，25(2): 107 .

[9] 湛欢, 刘大华. 参与式教学在卫生法学教学改革中的实践探索 $[J]$. 高等教育, 2016, $10(2): 70-71$.

[10］王国龙. 判决的可预测性与司法公信力 [J]. 求是学刊, 2014，41(1)：92-99.

[11] 马颖章. 法学实践教学体系构建的原则 $[\mathrm{J}]$. 继续教育研究, 2013，12：146-148. 
[12] 蔡枫瑜. 案例教学法在卫生法课程教学中的应用研究 [J]. 开封教育学院学报, 2015, $35(10)$ : 154-155.

\section{References}

[1] Meng Wan, Xiaohui Li. The Research of Case Method [J]. China University Teaching, 2014, 3:73-79.

[2] Robert Stevens. Law School: Legal Education in America from the1850s to the 1980s [M]. New Jersey: The Law Book Exchance, 2001: 68.

[3] Bruce A. Kimball, Young Chiristopher Langdell, 1826-1854: Theformation of an Educational Reformer [J]. Legal education, 2002, 56(1):189.

[4] Yi Wei, Jia He. The Reshape and Improvement of Case Study for National Health Law Teaching Reform [J]. Medicine \& Philosophy ((Humanistic \& Social Medicine Edition), 2010, 31(1):63-64.,

[5] Dongchu Tang, on the position and function of "case law" in the teaching of legal cases [J]. Journal of Hunan Political-legal Cadre College, 2002, 18(5): 108-109.

[6] Weimin Ren. Application of case teaching method in the teaching of medical law [J]. China Education Innovation Herald, 2009, NO.31: 80.

[7] Li Su. Institutional Analysis of Chinese legal skill education [J]. Jurists Review, 2008(2): 35.

[8] Lizhen Sun, Shuqin Wang. Organization mode and carrier analysis of interdisciplinary research in Research Universities [J]. R\&D Management, 2013, 25(2) 107.

[9] Huan Zhan, Dahua Liu. The practice of Participatory Teaching method in the teaching reform of health law [J]. Higher Education, 2016, 10(2):70-71.

[10]Guolong Wang. Predictability of judgment and judicial credibility [J]. Seeking Truth, 2014, 41(1): 92-99.

[11] Yingzhang Ma. Principles of construction of legal practice teaching system [J]. Continue Education Research, 2013, 12: 146-148.

[12]Fengyu Cai. Study on the application of case teaching method in the teaching of health law [J]. Journal of Kaifeng Institute of Education, 2015, 35(10): 154-155.

第一作者简介: 杨琳 (1982一)，女，湖北丹江口市，讲师，主要研究方向：卫生法, E-mail: $5810030 @ q q . c o m ;$

通讯作者简介: 郑玄 (1984一), 女, 湖北十堰, 讲师, 主要研究方向: 卫生法, E-mail: 826725887@qq.com. 